This is the final peer-reviewed accepted manuscript of:

Bonafede, A., Stuardi, C., Savini, F., Vazza, F., \& Brüggen, M. (2018). Constraining magnetic fields in galaxy clusters. Proceedings of the International Astronomical Union, 14(A30), 299-302.

The final published version is available online at: https://doi.org/10.1017/S1743921319004459

Rights / License:

The terms and conditions for the reuse of this version of the manuscript are specified in the publishing policy. For all terms of use and more information see the publisher's website. 


\title{
Constraining magnetic fields in galaxy clusters
}

\author{
Annalisa Bonafede ${ }^{1,2,3}$ Chiara Stuardi ${ }^{1,2}$, Federica Savini ${ }^{3}$, Franco \\ Vazza $^{1,2,3}$, and Marcus Brüggen ${ }^{3}$ \\ ${ }^{1}$ Dipartimento di Fisica e Astronomia, Università di Bologna, via P. Gobetti 93/2, 40129, \\ Bologna, Italy. \\ ${ }^{2}$ INAF - Istituto di Radioastronomia, Via Gobetti 101, I-40129 Bologna, Italy. \\ ${ }^{3}$ Hamburger Sternwarte, Universität Hamburg, Gojenbergsweg 112, 21029, Hamburg, \\ Germany. \\ email: annalisa.bonafede@unibo.it
}

\begin{abstract}
Magnetic fields originate small-scale instabilities in the plasma of the intra-cluster medium, and may have a key role to understand particle acceleration mechanisms. Recent observations at low radio frequencies have revealed that synchrotron emission from galaxy clusters is more various and complicated than previously thought, and new types of radio sources have been observed. In the last decade, big step forwards have been done to constrain the magnetic field properties in clusters thanks to a combined approach of polarisation observations and numerical simulations that aim to reproduce Faraday Rotation measures of sources observed through the intra-cluster medium. In this contribution, I will review the results on magnetic fields reached in the last years, and I will discuss the assumptions that have been done so far in light of new results obtained from cosmological simulations. I will also discuss how the next generation of radio instruments, as the SKA, will help improving our knowledge of the magnetic field in the intra-cluster medium.
\end{abstract}

Keywords. Galaxy clusters, magnetic field, non-thermal phenomena

\section{Introduction}

Galaxy clusters host magnetic fields that are responsible for a variety of phenomena on a large range of spatial scales. The intra-cluster medium (ICM) is an almost perfect plasma, and the magnetic field originates small-scale instabilities that can amplify the field itself and modify the ICM microphysics. On Mpc scale, the magnetic field interacts with Cosmic Ray electrons (CRe) and produce diffuse emission such as radio halos, mini halos, and radio relics. Recent observations with the LOw Frequency ARray (LOFAR, van Haarlem, et al. 2013) have shown that the radio emission in the ICM is more complex than initially thought. In particular, Savini et al. (2018) have discovered that steepspectrum emission on Mpc scale is observed also in clusters that do not show signs of major merger. In Fig. 1 (right panel), the radio emission from the galaxy cluster RXCJ1720.1+2638 is shown. This cluster is known to host a mini halo confined between two cold fronts, i.e. discontinuities in the X-ray surface brightness profile. It has been proposed that the cold fronts are formed during a minor merger, where the dense core of the cluster is perturbed and starts a sloshing motion in the dark matter potential well. LOFAR reveals that the radio emission extends well beyond the cold fronts. The radio emission inside and outside the core can be well separated both in brightness and in spectral index. The core emission (mini halo) is relatively flat $(\alpha \sim-1)$ and the spectral index distribution is uniform. The emission SW and NE of the core emission, beyond the cold fronts, is steeper $(\alpha \sim-2)$, suggesting either a different acceleration process and/or 
a different magnetic field strength in the two regions.

New emission has also been observed by LOFAR in the cluster MACSJ0717.0+3745 (Bonafede et al. 2018). This massive $\left(M_{500} \sim 1.1 \times 10^{15} M_{\odot}\right)$ high-z $(\mathrm{z}=0.546)$ cluster was known to host diffuse radio emission visible at $\mathrm{GHz}$ frequencies, which is not co-spatial with the X-ray emission from the gas. The radio emission is confined in the Eastern part of the cluster. LOFAR observations at $150 \mathrm{MHz}$ have confirmed this asymmetry in the radio emission (see Fig. 1, left panel), and a new radio arc with a linear size of $\sim 1.7$ $\mathrm{Mpc}$ has been discovered at the NW of the cluster centre. The analysis of the power spectrum of the gas density fluctuations - performed following the method by Zhuravleva et al. (2014) - has shown that there is a correlation between the amplitude of the power spectrum and the presence of radio emission. This might indicate a higher ratio of kinetic versus thermal energy in the region with radio emission than in the region without.

Recently, theoretical studies have focussed on the role of magnetic fields to accelerate particles in the cluster outskirts. Radio relics are linked to low Mach number shocks, that have a low acceleration efficiency (e.g. Brüggen et al. 2011). Shock waves should also amplify the magnetic field, but only the magnetic field amplification in the relic of the Coma cluster has been analysed so far (Bonafede et al. 2013) and no net amplification in the shocked region has been found.

\section{Constraints on magnetic fields and assumptions}

The analysis of the Faraday effect from sources inside and in the background of clusters is the most used technique to constrain the magnetic field. Using this method, Bonafede et al. (2010) used the Rotation Measure (RM) of 7 sources in the background of the Coma cluster and the Faraday code (Murgia et al. 2004), and derived that the magnetic field strength in the cluster centre is $B_{0} \sim 5 \mu \mathrm{G}$, with a radial profile that scales with the square-root of the gas density profile (i.e. $B(r) \propto n^{0.5}$ ). To derive these values, assumptions need to be made on the magnetic field properties. In particular, the magnetic field is assumed to be a Gaussian random field, characterised by a single power law power spectrum. Thanks to the increasing resolution reached by recent cosmological simulations, it is now possible to compare observed and simulated RM. Using an adaptive
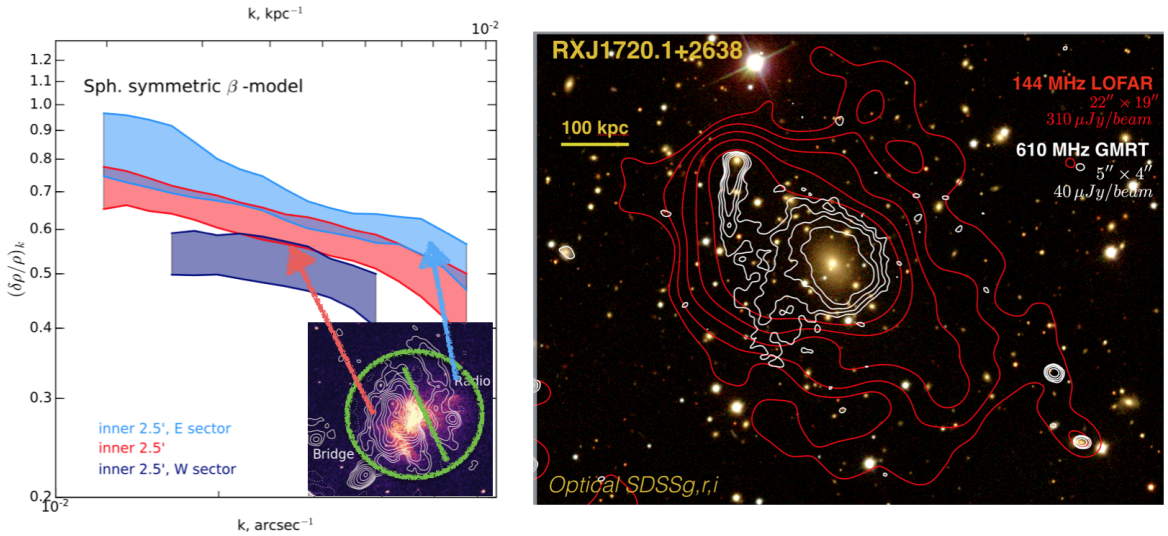

Figure 1. Left: power spectrum of the gas density fluctuations in the cluster MACSJ0717 in the regions with and without radio emission, as shown in the inset (Bonafede et al. 2018). Right: The cluster RXCJ1720.1+2638: optical emission and radio emission from GMRT at $610 \mathrm{MHz}$ (white) and LOFAR at $150 \mathrm{MHz}$ (red), from Savini et al (2018, in press). 
mesh refinement method, Vazza et al. (2018) have performed MHD cosmological simulations reaching a resolution of $\sim 4 \mathrm{kpc}$ in the cluster centres. A primordial magnetic field $B_{i}=0.1 \mathrm{nG}$ at $\mathrm{z}=30$ is evolved, and its amplification is observed to be above the one predicted from a pure adiabatic compression. As the resolution increases, the magnetic field amplification increases as well, reaching $B \sim \mu \mathrm{G}$ in the cluster centres. The magnetic field components show a departure from a Gaussian distribution, with a tail towards high values of the magnetic field. The amplitude of this tail depends on time, cluster dynamics, and resolution of the simulation. The net effect is that the RM profile for a Coma-like cluster can be reproduced with slightly lower values of $B_{0}$ than those derived by Bonafede et al. (2010). In the specific case analysed by Vazza et al. (2018), the average value of the magnetic field in the core is $B_{\text {core }} \sim 1.5 \mu \mathrm{G}$.

Cosmological simulations also predict that magnetic field power spectrum deviates from a simple power law. The relevant scales to understand the role of magnetic field in accelerating particles would be the injection scale $\left(L_{i n j} \sim\right.$ few $\left.100 \mathrm{kpc}\right)$ and the viscous scale $\left(L_{v i s c} \sim\right.$ few kpc, or even lower if the Spitzer viscosity is suppressed in the ICM). The power spectrum can be approximated by a power law between $L_{i n j}$ and $L_{v i s c}$ (see e.g. Ryu, this meeting, Xu et al. 2009).

However, it must be noted that the number of free parameters would increase if nonGaussian magnetic fields and non power law power spectra were considered. The constraints on the magnetic fields that have been obtained so far are based on few sources per cluster (from 1 to 7 ), and the modelling done by the authors to reproduce mock RM images already have a total of 5 free-parameters. Hence, we conclude that - despite more complicated models for the magnetic field should be ideally considered - the limiting factor is the low number of sources per cluster. Considering more complicated models of magnetic field and increasing the number of free parameters will make sense only when future observations will be able to sample the RM through several tens of lines on sight per cluster.

\section{New results with present instruments and future perspectives}

The advent of spectro-polarimetric and wide-band receivers enables us to make an important step forward in the study of cluster magnetic fields. We started a project to constrain the magnetic field amplification by low Mach number shocks in clusters. To

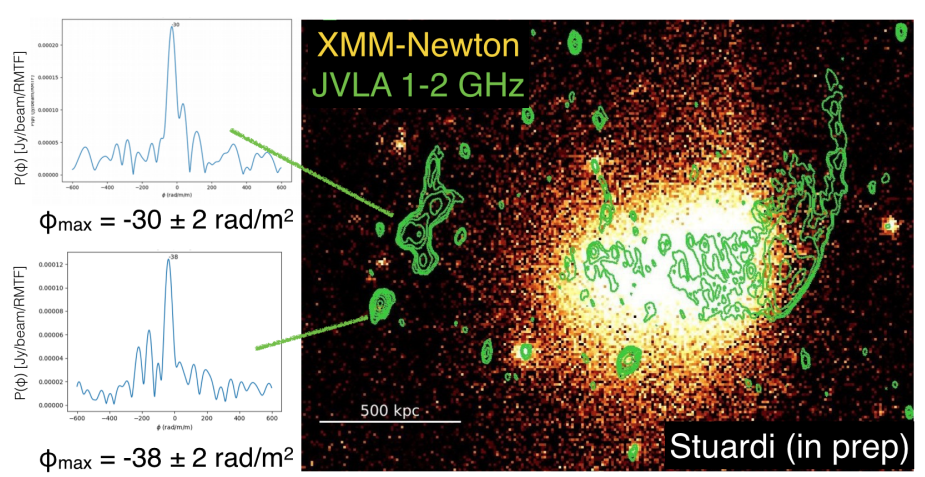

Figure 2. Radio emission from the cluster RXCJ1314 at 1-2 GHz (Stuardi et al, in prep.). The left panels show the Faraday spectra through one source in the relic (top) and in the pre-shock region (bottom). The Galactic foreground RM is $\phi \sim-30 \mathrm{rad} / \mathrm{m}^{2}$, consistent with the values found for the two sources. 
overcome the limit of few sources observed per cluster, we built up a sample of cluster with double relics, and obtained $\sim 80$ h observing time at the Jansky Very Large Array. In Fig. 2, we show the cluster RXCJ1314-2515 (Stuardi et al in prep). We analysed the Faraday spectrum of the emission using the Rotation Measure synthesis technique (Brentjens \& de Bruyn 2005). So far, the regions that we have analysed seem consistent with the Galactic RM, suggesting little/no amplification of the magnetic field in the shocked region.

The next generation of radio instruments, i.e. the Square Kilometer Array (SKA) will permit a study of the magnetic field in individual clusters. The expected number density of polarised sources that SKA will observe is $\sim 300$ per square degree(Rudnick \& Owen 2014). This means that we will be able to study the magnetic field in a Coma-like cluster using $\sim 50$ radio sources, i.e. a factor 7 higher than present-day studies. Bonafede et al. (2015) have analysed the RM grid that SKA will provide for clusters of different mass, finding that we will have enough sources to constrain the magnetic field in the background of low-mass galaxy clusters and galaxy groups. Having such a high number of RM samples through the line of sight, one may be able to investigate different configurations for the magnetic field, i.e. non-Gaussian distributions of the components and different functions for the power spectrum.

\section{Conclusions}

Obtaining constraints on the magnetic field in the ICM is crucial to understand particle acceleration mechanisms and the ICM microphysics. One must bear in mind that magnetic fields are not directly observable, and the constraints derived through observations rely on the assumptions that have been made. State-of the art instruments, such as the JVLA are very promising to obtain statistical constraints on the magnetic field using samples of clusters. Using this approach, Stuardi et al (in prep) are already investigating for the first time the magnetic field amplification by low Mach number shocks in radio relics. In the next years, the advent of the SKA will permit a detailed study on individual clusters, and will in principle allow us to investigate different model assumptions for the magnetic field structure.

Acknowledgments: AB and CS acknowledge financial support from the ERC-Stg DRANOEL, no 714245. FV acknowledges financial support from the ERC-Stg MAGCOW, no.714196.

\section{References}

Bonafede A., et al., 2018, MNRAS, 478, 2927

Bonafede A., et al., 2015, aska.conf, 95

Bonafede A., Vazza F., Brüggen et al. 2013, MNRAS, 433, 3208

Bonafede A., Feretti L., Murgia M., Govoni et al. 2010, A\&A, 513, A30

Brentjens M. A., de Bruyn A. G., 2005, A\&A, 441, 1217

Brüggen M., Bykov A., Ryu D., Röttgering H., 2011, SSR, p. 138

Murgia M., Govoni F., Feretti L., et al., 2004, A\&A, 424, 429

Rudnick L., Owen F. N., 2014, ApJ, 785, 45

van Haarlem M. P., Wise M. W., Gunst A. W., Heald G., et al 2013, A\&A, 556, A2

Savini F., et al., 2018, MNRAS, 478, 2234

Vazza F., Brunetti G., Brüggen M., Bonafede A., 2018, MNRAS, 474, 1672

Xu H., Li H., Collins D. C., Li S., Norman M. L., 2009, ApJ, 698, L14

Zhuravleva I., Churazov E. M., Schekochihin A. A., et al. 2014, ApJ, 788, L13 